\title{
HALO FORMATION IN INTENSE BUNCHED BEAMS *
}

\author{
$\underline{\text { A.V. Fedotov }}^{\dagger}$ and R.L. Gluckstern \\ Physics Department, University of Maryland, College Park, MD 20742
}

\section{Abstract}

The latest designs for high current ion linacs (Accelerator for the Transmutation of Waste, Accelerator for the Production of Tritium, Heavy Ion Drivers, Spallation Neutron Source Injector) require minimal radioactivation by the beam striking the beam pipe. As a result, efforts are being made to understand and control the growth of beam halo. There is general agreement that halos develop as a result of the parametric resonance between the ion oscillations in the beam bunch and collective oscillations of the bunch itself induced by mismatch in the linac. Analytic studies for a 2-D KV beam were found to give excellent agreement with corresponding computer simulations, which were then extended to other 2-D beams. Recently, analytic and numerical studies were performed for 3-D beam bunches (6-D phase space distributions), focusing attention on the formation of longitudinal halos and the possibility of bunch growth or loss of longitudinal bunch stability, as well as coupling between the longitudinal and transverse halos.

\section{INTRODUCTION}

The need for high current in a variety of new accelerator applications has focused a great deal of attention on understanding the phenomenon of halo formation in ion beams, which can cause excessive radiation of the accelerator. This understanding requires both an analytical model which explains available observations as well as computer simulations to verify both the assumptions of the model and its predictions.

Analytic models have been developed to study halo development in both 2-D beams and 3-D beam bunches in a linac. These models suggest that the most likely explanation for the halos which have been observed and which are likely to be seen in future high current linacs involves the parametric resonance between the collective modes which describe "breathing" and the motion of individual ions. When these models are used in conjunction with multiparticle simulations involving millions of particles, which are now practical with supercomputers and parallel processing, one can have great confidence in the predictions for halo formation and emittance growth which are so crucial for the designs of high current acceleration of short beam bunches.

\footnotetext{
${ }^{*}$ Work supported by the U.S. Department of Energy

$\dagger$ fedotov@physics.umd.edu
}

\section{2-D MODEL}

Early attention was devoted to the analytic study of 2-D round beams in a continuous focusing channel. In particular, the KV distribution [1], a hyperspherical shell in the 4-D phase space with the self-consistent [2] distribution

$$
f(H)=N \delta\left(H_{0}-H\right)
$$

where

$$
H=\frac{m v^{2}}{2}+\frac{k r^{2}}{2}+e \Phi_{s c}(\boldsymbol{r})
$$

had the useful features of a uniform charge density within the beam, and uniform density in the $x$ and $y$ phase space projections. Here $H_{0}$ and $N$ are constants, $k$ is the constant external focusing gradient, and $e \Phi_{s c}(\boldsymbol{r})$ is the potential energy at $r$ due to space charge.

Use of the equation for the beam envelope [3] permitted the analytic description of a "breathing" beam, in which the charge density oscillated between too tight and too loose a match to the external focusing force. These oscillations provided a periodic force to the ion motion, which was simple harmonic as long as the ions remained inside the beam. But for ions which traveled beyond the beam boundary, the oscillations were non-linear. In this case the ion's nonlinear motion in the presence of a periodic force allowed it to be trapped in the parametric resonance, where the breathing frequency was twice the ion oscillation frequency. The analytic model thus predicted the formation of a "halo" [4] for certain combinations of mismatch and tune depression. The numerical simulations using the "particle-core" model confirmed the validity of the models, and pointed as well to the existence of chaotic motion as the tune depression became more severe [5]-[9].

Subsequent work focused on the possible mechanism for particles escaping from the beam into the region of nonlinear oscillation [10]. In addition, numerical simulations were run for other, more physical, self-consistent stationary distributions of the form

$$
f(\boldsymbol{r}, \boldsymbol{v})=N\left(H_{0}-H\right)^{n},
$$

with $n=0,1$ [11]. These simulations exhibited the same halo structure and phase space patterns seen for the KV distribution, but with somewhat different quantitative dependence on mismatch and tune depression. The localization of the halo radius to approximately the same value predicted by the KV distribution gave the linac designers confidence that a beam pipe wall could be placed far enough from the beam to avoid intercepting the halo particles. 


\section{3-D MODEL}

Attention then shifted to short 3-D beam bunches of ellispoidal shape with $c / a=$ length/width ratio in the range $2-4[12,13]$. We continued our effort to study the selfconsistent phase space stationary distributions of the form

$$
f(\boldsymbol{r}, \boldsymbol{v})=N\left(H_{0}-H\right)^{n},
$$

but this time, for $n=-1 / 2$, the differential equation for the charge density was linear and could be solved analytically [13]. In addition, for $c / a>2$, the "breathing" modes could be approximately separated into transverse and longitudinal modes, each of which was capable of generating a halo. Thus the picture was of a beam bunch which, when mismatched accordingly, generated either a transverse or a longitudinal halo, or both. The signature of the longitudinal halo was the same as that of the transverse halo (a "peanut diagram" in the phase space projection). The transverse and longitudinal mismatch and tune depression parameter space was extensively explored with numerical simulations [13]. But a new concern surfaced: Would the longitudinal halo permit the loss of ions from the rf bucket? Unfortunately, the bucket "walls" cannot be moved far away without increasing the length and cost of the linac. Details about effects of non-linear RF fields can be found in [14].

Other issues involving halo formation were looked at, including equipartitioned distributions which were rms matched but not self-consistent [15]. These involved a rapid initial phase space redistribution, leading to a relatively small change in the parameters and extent of the halo formation due to the mismatch. In addition, they also point to the presence of a transverse-longitudinal coupling which allows either kind of halo to develop from either a transverse or longitudinal mismatch [15].

\section{STATIONARY 6-D PHASE SPACE DISTRIBUTION}

\subsection{Analytic approximation to a spheroidal bunch}

We take for the azimuthally symmetric 6-D phase space distribution

$$
f(\boldsymbol{x}, \boldsymbol{p})=N\left(H_{0}-H\right)^{-1 / 2}
$$

where

$$
H=k_{x} r^{2} / 2+k_{z} z^{2} / 2+e \Phi_{s c}(\boldsymbol{x})+m v^{2} / 2
$$

Here $\boldsymbol{p}=m \boldsymbol{v}, r^{2}=x^{2}+y^{2}$, and $k_{x}, k_{z}$ are the smoothed transverse and longitudinal restoring force gradients. The quantity $\Phi_{s c}(x)$ is the electrostatic potential due to the space charge of the bunch. The distribution is normalized such that

$$
\int d \boldsymbol{x} \int d \boldsymbol{p} f(\boldsymbol{x}, \boldsymbol{p})=1
$$

The charge distribution corresponding to Eq. (5) is

$$
\begin{aligned}
\rho(\boldsymbol{x}) & =Q \int d \boldsymbol{p} f(\boldsymbol{x}, \boldsymbol{p}) \\
& =N Q m^{3} \int d \boldsymbol{v}\left[G(\boldsymbol{x})-\frac{m v^{2}}{2}\right]^{-1 / 2},
\end{aligned}
$$

where

$$
G(\boldsymbol{x}) \equiv H_{0}-\frac{k_{x} r^{2}}{2}-\frac{k_{z} z^{2}}{2}-e \Phi_{s c}(\boldsymbol{x}) .
$$

Performing the integral over $d \boldsymbol{v} \equiv v^{2} d v d \Omega_{v}$ in Eq. (8) leads to

$$
\rho(\boldsymbol{x})=Q G(\boldsymbol{x}) / \int d \boldsymbol{x} G(\boldsymbol{x}),
$$

where the normalization constant satisfies

$$
2 \sqrt{2} \pi^{2} N m^{3 / 2} \int d \boldsymbol{x} G(\boldsymbol{x})=1 .
$$

From Eq. (9) and Poisson's equation, we write

$$
\nabla^{2} G(\boldsymbol{x})=-k_{s}-e \nabla^{2} \Phi_{s c}=-k_{s}+\left(e / \epsilon_{0}\right) \rho(\boldsymbol{x})
$$

where

$$
k_{s}=2 k_{x}+k_{z} .
$$

Using Eq. (10), we obtain the partial differential equation for $G(\boldsymbol{x})$

$$
\nabla^{2} G(\boldsymbol{x})=-k_{s}+\kappa^{2} G(\boldsymbol{x})
$$

where

$$
\kappa^{2}=\left(e Q / \epsilon_{0}\right) / \int d \boldsymbol{x} G(\boldsymbol{x}) .
$$

The solution of Eq. (14) for an axisymmetric, spheroidal shaped bunch can most easily be written in the spherical coordinates $R, \theta$ for which

$$
z=R \cos \theta, r=R \sin \theta,
$$

as

$$
G(\boldsymbol{x})=\left(k_{s} / \kappa^{2}\right) g(\boldsymbol{x})
$$

where

$$
g(\boldsymbol{x})=1+\sum_{\ell=0}^{\infty} \alpha_{\ell} P_{2 \ell}(\cos \theta) i_{2 \ell}(\kappa R) .
$$

Here $P_{2 \ell}(\cos \theta)$ are the even (fore-aft symmetric) Legendre polynomials and $i_{2 \ell}(\kappa R)$ are the spherical Bessel functions (regular at $\kappa R=0$ ) of imaginary argument.

Since $g(\boldsymbol{x})$ is proportional to the charge density, the edge of the bunch is defined as the border $g(\boldsymbol{x})=0$, closest to the origin. We therefore choose the $\alpha_{\ell}$ 's so that the surface of the bunch reproduces, as closely as possible, the ellipsoidal surface.

We also note that $m\left\langle\dot{x}^{2}\right\rangle=m\left\langle\dot{y}^{2}\right\rangle=m\left\langle\dot{z}^{2}\right\rangle=m\left\langle v^{2}\right\rangle / 3$ because $H$ depends only on $v^{2}$ and $\boldsymbol{x}$. Thus our choice of a stationary distribution of the form $f(H)$ automatically corresponds to equipartition (equal average kinetic energy in the three spatial directions). 


\subsection{Numerical implementation}

We have developed a 3-D particle-in-cell (PIC) code HALO3D to test the analytic model described above, and to explore halo formation [13]. The single-particle equations of motion are integrated using a symplectic, split-operator technique [16]. The space charge calculation uses area weighting ("Cloud-in-Cell") and implements open boundary conditions with the Hockney convolution algorithm [17]. The code runs on parallel computers, and in particular, the space charge calculation has been optimized for parallel platforms using the Ferrell-Bertschinger method [18]. Some details about the code can be found in [19].

We initially populate the 6-D phase space according to Eq. (5), and then mismatch the $x, y, z$ coordinates by factors $\mu_{x}=\mu_{y}=1+\delta a / a, \mu_{z}=1+\delta c / c$ and the corresponding momenta by $1 / \mu_{x}=1 / \mu_{y}, 1 / \mu_{z}$, with $a, c$ being the minor and major semiaxes of our spheroidal bunch, respectively.

\subsection{Longitudinal halo}

We performed a systematic study for different $c / a$ and mismatch factors in the range of interest [20], by looking at the halo extent at the time when the beam comes to a roughly saturated state after the development of a halo. Our new result is the dependence of the halo extent on tune depression. One sees a significant increase in halo extent for severe tune depressions. In addition the halo extent clearly depends on the mismatch parameter. The approximately linear dependence of the halo extent on the mismatch factor $\mu$ indicates that a serious effort should be made to match the beam to the channel as accurately as possible.

Simulation results [13] show that the halo intensity (roughly defined as the fraction of particles outside the core in phase space) depends primarily on the mismatch. Severe mismatches lead to several percent of the particles in the halo, which is clearly outside acceptable limits. No significant dependence of halo intensity on the tune depression is seen. Also, for tune depression $\eta_{z} \leq 0.4$ the clear peanut diagram in the longitudinal phase space now has a chaotic behavior.

One more important feature is how fast the halo develops. We first make the observation that for comparable mismatches the longitudinal halo develops much faster than the transverse halo when the mismatches and/or tune depressions are not severe. Such behavior simply occurs because for fixed charge we have $\eta_{z}<\eta_{x}$ for elongated equipartitioned bunches. For severe mismatches and/or tune depressions both the longitudinal and transverse halos develop very quickly. Of particular interest is the clear dependence of halo onset on tune depression. Specifically, for more severe tune depression the halo starts to develop earlier. More details can be found in [13].

\subsection{Transverse halo}

The transverse halo closely duplicates all the features observed for non-linear stationary distributions in 2-D simulations [11]. The agreement between 2-D and 3-D simulations is very good. The only two differences seen are related to the onset/rate of halo development. In the recent 3-D simulations there is a clear dependence on the tune depression which was not the case in the corresponding 2-D simulations [11]. The second difference is that the transverse halo in the 3-D simulations develops significantly faster than in 2-D for comparable mismatches and tune depressions. More details can be found in [13].

\subsection{Coupling effects}

In performing 3-D simulations we encounter halo formation in a beam bunch, where we clearly see coupling between the longitudinal and transverse motion. It was already noted [13] that due to the coupling between $r$ and $z$, a transverse or longitudinal halo is observed even for a very small mismatch (less than $10 \%$ ) as long as there is a significant mismatch in the other plane. Further numerical investigation [15] of this question showed that the effect of coupling becomes extremely important for nearly spherical bunches $(c / a \leq 2)$ which is typical of the parameter range of interest for the APT design [20]. For example, for the short bunch with $c / a=2$, with only a longitudinal initial mismatch ( $\mu_{z}=1.5, \mu_{x}=\mu_{y}=1.0$ ), one finds particles at large amplitude in both the longitudinal and transverse directions [15].

\section{NON-STATIONARY 6-D PHASE SPACE DISTRIBUTION}

After we established the parameters which lead to halo formation in 3-D beam bunches for the self-consistent 6-D phase space stationary distribution [13], we explored rms matched distributions which are not self-consistent, to determine the extent to which the relatively rapid redistribution of the 6-D phase space contributes to the formation of halos [15].

\subsection{Stability of the matched distribution}

We have shown that an rms matched 3-D beam can produce transverse and/or longitudinal halos for a wide range of space charge intensity even when it is initially perfectly matched. Of course, from a practical point of view such halos are not important because the halo extent is very small for the mismatch factor $\mu=1.0$ (the detailed study of the halo extent on a mismatch factor was presented in $[13,15])$.

The redistribution process in a non-stationary beam with initial zero mismatch causes the core to perform an oscillation about its initial distribution which is equivalent to introducing a relatively small mismatch for the stationary distributions. The important consequence is that the redistribution process by itself (zero initial rms mismatch) does 
not lead to significant emittance growth [15].

\subsection{Initially mismatched beam}

Numerical 3-D simulations with the initially mismatched non-stationary distributions [15] confirmed all the characteristics of halos observed for the stationary distribution [13]. The main difference is that for a non-stationary distribution the halo extent is larger (especially for the Gaussian) than the halo extent of the stationary distribution with the same initial mismatch parameters.

\section{HALO FORMATION IN A PERIODIC FOCUSING CHANNEL}

The halo properties in periodically focused cases have been also extensively studied [21]-[27]. Apart from the instabilities due to the structure-driven resonances, these studies showed a close resemblance to the continuous focusing channel results.

\section{OTHER ISSUES INVOLVING HALO FORMATION}

\subsection{Coulomb scattering}

Various mechanisms can potentially cause beam halo. Some recent studies suggested that Coulomb collisions in the beam bunch can contribute significantly to beam bunch growth and halo development in linear accelerators. Despite the general belief that collisions are not important it is clear that a rigorous treatment of this question is needed. In an effort to explore this issue in detail we have undertaken an analysis of the effects of Coulomb scattering between ions in a self-consistent spherical bunch [28].

We have calculated the effect of single Coulomb scattering of a self-consistent 6-D distribution for a spherical beam bunch. In this calculation we found that single collisions are capable of populating a thin spherical shell around the beam bunch. This result is for the stationary phase space distribution with $n=-1 / 2$, but it is probably quantitatively similar for other higher values of $n$. When the beam is non-equipartitioned or the beam with the stationary distribution is rms mismatched, the thickness of the shell can be significantly larger, depending on the equipartitioning factor. However, the rate of this process is very small. For the relatively singular distribution with $n=-1 / 2$, a proton bunch with a normalized emittance $\epsilon_{N} \sim 10^{-6}[\mathrm{~m}$ $\mathrm{rad}$ ] and a radius of $1 \mathrm{~cm}$ will populate the shell with a probability of $10^{-11}$ per kilometer of linac. For distributions with $n>0$, this rate of population is further reduced by a factor $10^{-4}$

Our conclusion is that the effect of single Coulomb collisions on halo development in high current ion linear accelerators is not important. A similar analysis for nonstationary distributions was performed by N. Pichoff [29], who arrived at the same conclusions.
We then related our analysis to diffusion caused by many small angle Coulomb collisions, with the conclusion that the effect of multiple Coulomb collisions in halo development in high current ion accelerators is also expected not to be important [28].

\subsection{Halo formation in circular accelerators}

Space-charge can also lead to emittance growth and halo generation in circular accelerators. In undertaking a study of the space-charge dynamics in high intensity rings one needs to consider two different effects.

The first one is associated with the intrinsic halo formation due to the core mismatch, corresponding to a parametric resonance of the coherent frequency with twice the incoherent depressed tune of individual ions. Although the tune depression in the ring is very small compared with high intensity linacs this effect will still generate a halo of significant size around the beam core.

The second effect is associated with the machine resonances due to the magnet imperfections, and is governed by the resonant effect of different coherent mode frequencies [30]-[31].

\section{SUMMARY}

Analytic models have been developed to study halo development in both 2-D beams and 3-D beam bunches in a linac. The detailed study requires both an analytical model which explains available observations as well as computer simulations to verify both the assumptions of the model and its predictions.

Our recent contributions to these efforts have been the construction of a model which identifies a major mechanism for transverse halo formation [4], followed by the construction of a self-consistent 6-D phase space distribution for studies of halo formation in spheroidal bunches in a linear external confining field [13]. What we found [4] is that halo formation appears to arise from a parametric resonant coupling of individual particle oscillations with collective oscillation of the charged bunch, primarily those describing the breathing modes. We explored the dependence of the halo properties (extent, rate of growth, intensity, etc.) on the longitudinal and transverse rms tune depressions and mismatches [13]. These studies automatically assumed equipartition of kinetic energy between the longitudinal and two transverse directions. We then performed numerical studies with an rms matched, but otherwise non-self-consistent 6-D distribution [15]. In these studies we found that starting with a non-self-consistent distribution altered the parameters for halo formation obtained for the self-consistent distribution only slightly.

Our main conclusion is that the longitudinal halo in a linac is of great importance. The control of the longitudinal halo could be challenging if the phase width of a beam bunch in the RF bucket cannot be made sufficiently small. 


\section{REFERENCES}

[1] I.M. Kapchinsky, Theory of Resonance Linear Accelerators, Harwood Academic Publishers, New York, 1985.

[2] Any distribution which is only a function of the Hamiltonian will be self-consistent.

[3] M. Reiser, Theory and Design of Charged Particle Beams, Wiley, New York (1994).

[4] R.L. Gluckstern, Phys. Rev. Letters 73, 1247 (1994).

[5] J.S. O'Connell, T.P. Wangler, R.S. Mills, and K.R. Crandall, in Proceedings of the 1993 Particle Accelerator Conference (IEEE, Washington, DC, 1993), p. 3657.

[6] R.A. Jameson, in 'Frontiers of Accelerator Technology' (Proceed. of the Joint US-CERN-Japan Intern'l School, 1994), World Scient., Singapore (1996).

[7] J.M. Lagniel, Nucl. Inst. Meth. A345 (1994) 46; A345 (1994) 405.

[8] A. Riabko, M. Ellison, X. Kang, S.Y. Lee, D. Li, J.Y. Liu, X. Pei, and L. Wang, Phys. Rev. E 51, 3529 (1995).

[9] H. Okamoto and M. Ikegami, Phys. Rev. E 55, 4694 (1997).

[10] R.L. Gluckstern, W-H. Cheng, and H. Ye, Phys. Rev. Letters 75, 2835 (1995); R.L. Gluckstern, W-H. Cheng, S.S. Kurennoy and H. Ye, Phys Rev. E 54, 6788 (1996).

[11] R.L. Gluckstern and S.S. Kurennoy, in Proceedings of the 1997 Particle Accelerator Conference, Vancouver, Canada (IEEE, Piscataway, NJ, 1998), p. 1950.

[12] J.J. Barnard and S.M. Lund, in Proceedings of the 1997 Particle Accelerator Conference, Vancouver, Canada (IEEE, Piscataway, NJ, 1998), p. 1929; S.M. Lund and J.J. Barnard, ibid., p. 1932.

[13] R.L. Gluckstern, A.V. Fedotov, S. Kurennoy and R. Ryne, Phys. Rev. E 58, 4977 (1998).

[14] J.J. Barnard, S.M. Lund and R.D. Ryne, in Proceedings of the 1998 Linear Accelerator Conference, Chicago, IL (1998).

[15] A.V. Fedotov, R.L. Gluckstern, S.S. Kurennoy and R.D. Ryne, Phys. Rev. ST Accel. Beams, 2, 014201 (1999).

[16] E. Forest, J. Bengtsson, and M.F. Reusch, Phys. Lett. A 158, 99 (1991).

[17] R.W. Hockney and J.W. Eastwood, Computer Simulation Using Particles, Adam Hilger, NY (1988).

[18] R. Ferrell and E. Bertschinger, Int. J. Mod. Phys. C 5, 933 (1994).

[19] R. Ryne, S. Habib, J. Qiang, K. Ko, Z. Li, B. McCandless, W. Mi, C. Ng, M. Saparov, V. Strinivas, Y. Sun, X. Zhan, V. Decyk, G. Golub, in Proceedings of the 1998 Linear Accelerator Conference, Chicago, IL (1998).

[20] APT Conceptual Design Report, Los Alamos Report No. LA-UR-97-1329, 1997.

[21] C. Chen and R.C. Davidson, Phys. Rev. E 49, 5679 (1994).

[22] C. Chen and R.C. Davidson, Phys. Rev. Lett. 72, 2195 (1994).

[23] Q. Qian, R.C. Davidson and C. Chen, Phys. Plasmas 1, 3104 (1994).

[24] S.Y. Lee and A. Riabko, Phys. Rev. E 51, 1609 (1995).
[25] Q. Qian and R.C. Davidson, Phys. Rev. E 53, 5349 (1996).

[26] M. Ikegami and H. Okamoto, Jpn. J. Appl. Phys. 36, 7028 (1997).

[27] M. Pabst, K. Bongardt and A. Letchford, in Proceedings of the 1998 EPAC (Stockholm, Sweden, 1998), p. 146.

[28] R.L Gluckstern and A.V. Fedotov, Coulomb Scattering Within a Spherical Beam Bunch in a High Current Linear Accelerator, these Proceedings; submitted for publication in Phys. Rev. ST Accel. Beams, 1999.

[29] N. Pichoff, DAPNIA/SEA Report 98-46, France (1998).

[30] R.L. Gluckstern, in Proceedings of the 1970 Linear Accelerator Conference, edited by M.R. Tracy (Fermilab, Batavia, IL), p. 811.

[31] R. Baartman, AIP Conference Proceeding 448, edited by A.U. Luccio and W.T. Weng (AIP, N.Y., 1998), p. 56; and references therein. 